\title{
MODULES OVER POLYCYCLIC GROUPS HAVE MANY IRREDUCIBLE IMAGES
}

\author{
by KENNETH A. BROWN
}

(Received 16 October, 1979)

1. Introduction. Recall that a Noetherian ring $R$ is a Hilbert ring if the Jacobson radical of every factor ring of $R$ is nilpotent. As one of the main results of [13], J. E. Roseblade proved that if $J$ is a commutative Hilbert ring and $G$ is a polycyclic-by-finite group then $J G$ is a Hilbert ring. The main theorem of this paper is a generalisation of this result in the case where all the field images of $J$ are absolute fields-we shall say that $J$ is absolutely Hilbert. The result is stated in terms of the (Gabriel-Rentschler-) Krull dimension; the definition and basic properties of this may be found in [5]. Let $M$ be a finitely generated right module over the ring $R$. We write $A n_{R}(M)$ (or just $A n n(M)$ ) for the ideal $\{r \in R: M r=0\}$, the annihilator of $M$ in $R$. If $M$ is also a left module, its left annihilator will be denoted $1-\mathrm{Ann}_{R}(M)$. If $R$ is a group ring $J G$, put

$$
T_{G}(M)=\bigcap\left\{\mathrm{Ann}_{R}(X): X \text { an irreducible image of } M\right\} .
$$

Let $\mathrm{k}-\operatorname{dim}(M)$ denote the Krull dimension of $M$.

THEOREM A. Let $M$ be a finitely generated $J G-m o d u l e$, where $J$ is an absolutely Hilbert domain and $G$ is a polycyclic-by-finite group. Let $I=\operatorname{Ann}_{J G}(M)$. Then

$$
\mathrm{k}-\operatorname{dim}(J G / I)=\mathrm{k}-\operatorname{dim}\left(J G / T_{G}(M)\right) \text {. }
$$

Roseblade has observed that, even when $J$ is a field and $G$ is finite, we cannot in general conclude that $I=T_{G}(M)$ in the above situation $[13, \S 5.4]$. If, however, $I$ is a prime ideal of $J G$ then Theorem A implies that $I=T_{G}(M)$; in particular, on taking $M$ to be a prime factor ring, we retrieve the conclusion that $J G$ is a Hilbert ring.

Suppose that $M$ is a critical $J G$-module-that is, a module whose proper factors have strictly smaller Krull dimension. Then, as the main result of [16], D. Segal proved that, provided $G$ is actually nilpotent-by-finite and $\mathrm{k}-\operatorname{dim}(J) \leq 1, M$ is residually simple; that is,

$$
0=\bigcap\{K: K \subseteq M, M / K \text { irreducible }\} .
$$

It remains an open question whether Segal's theorem is true when $G$ is polycyclic-byfinite. While Theorem A certainly provides evidence in favour of this possibility, there does not appear to be any obvious route from Theorem $\mathrm{A}$ to the desired conclusion.

Return once more to the notation of Theorem $\mathrm{A}$, and suppose that $H$ is a nilpotent normal subgroup of $G$ such that the augmentation ideal $\mathfrak{h}$ of $J H$ is contained in $T_{G}(M)$. Then Roseblade proved [13, Theorem B] that there exists $n \geq 1$ such that $\mathfrak{h}^{n} \subseteq I$. He asked whether this conclusion remained valid if $\mathfrak{h}$ were replaced by an arbitrary ideal of $J H[13$, p. 310]. We answer this question with the next theorem.

Glasgow Math. J. 22 (1981) 141-150. 
THEOREM B. In the notation of Theorem $A$, if $K$ is an ideal of $J G$, with $I \subseteq K \subseteq$ $T_{G}(M)$, and $K / I$ has the $A R$ property in $J G / I$ then there exists $n \geq 1$ such that $K^{n} \subseteq I$.

For the definition of the AR property, see $\$ 3$. We indicate in Corollary $\mathrm{C}$ why the above result yields a positive answer to Roseblade's question.

As a consequence of his theorem, Roseblade deduced that if $H$ is any normal subgroup of $G$ whose augmentation ideal annihilates every chief factor of $M$ then $M \mathfrak{h}^{n}=0$ for some $n \geq 1[13$, Corollary B]. We extend this result from augmentation ideals to arbitrary ideals of $J G$ (Corollary D).

The paper is organised as follows. In $\$ 2$ we obtain a generalisation of yet another result of Roseblade [13, Theorem E]. This result (Theorem 2.1) is needed in the proofs of Theorems A and B, and may be of some independent interest. In its present form, Theorem 2.1 is due to Dan Segal (unpublished). (We are grateful to him for supplying this improvement of an earlier version of the result.) After obtaining some preliminary lemmas in \$3, we prove the main results in \$4. It is necessary to prove Theorems A and B together, arguing by induction on the Hirsch number of the group $G$. The argument makes use of the results of $[2,86]$, which describe the meet irreducible factor rings of $J G$, to reduce the proof to the case where the annihilator $I$ of $M$ is a prime ideal, and the group $G$ has various desirable properties. Heavy use is then made of the main result of [14] to deduce Theorems A and B from Theorem 2.1. Finally, \$5 contains a few concluding remarks. In particular, we note there that Theorem A cannot be extended to arbitrary non-commutative Hilbert rings.

2. A generalisation of a result of Roseblade. The statement and proof of the result stated below involves the concept of a plinth $A$ for the polycyclic-by-finite group $G$. Our use of this term follows $[14,82.3]$. Namely, let $A$ be a finitely generated Abelian group, and $G$ a polycyclic-by-finite group acting on $A$. Then $A$ is a plinth for $G$ if and only if the $\mathbb{Q} G$-module $A \otimes_{\mathbf{Z}} \mathbb{Q}$ is an irreducible $\mathbb{Q} H$-module for every subgroup $H$ of finite index in $G$. The plinth $A$ is eccentric if and only if $G / C_{G}(A)$ is infinite. A non-empty subset $X$ of $A$ is said to be $G$-orbital if it has only finitely many $G$-conjugates.

Let $I$ be an ideal of the group ring $R G$. We write $I^{+}=\{g \in G:(g-1) \in I\}$. Thus $I^{+}$is a normal subgroup of $G$. When $I^{+}=1, I$ is said to be faithful. We use the appropriate small German letters to denote augmentation ideals; thus, if $H$ is a subgroup of $G$, we write $\mathfrak{h}=\sum_{\mathfrak{h} \in \boldsymbol{H}}(h-1) R H$. If $H \subseteq I^{+}$then $\mathfrak{h} G \subseteq I$. If $P$ is an ideal of $R H$ then the normaliser of $P$ in $G$ is $N_{G}(P)=\left\{g \in G: P^{\mathrm{g}}=P\right\}$.

The following result generalises [13, Theorem E].

THEOREM 2.1 (D. Segal). Let $G$ be a polycyclic-by-finite group, let $A$ be a finitely generated Abelian group on which $G$ acts, and let $J$ be an absolutely Hilbert domain. If $0 \neq \lambda \in J A$ then there exists a maximal ideal $M$ of $J A$ such that $M$ contains no $G$-conjugate of $\lambda$.

Proof. Since $J$ is semisimple, there is a maximal ideal $P$ of $J$ such that $\lambda \notin P A$. Hence, replacing $J$ by $J / P$, we may assume that $J=k$ is an absolute field. Furthermore, replacing 
$k$ by the finite field generated by the coefficients of $\lambda$, we may assume that $k$ is finite, as in $[13$, p. 321]. We argue by induction on the rank $h(A)$ of $A$. If $A$ is a plinth (and so if $h(A)=1)$ then the result is $[13$, Theorem E]. Suppose that $A$ is not a plinth, so that by [13, Lemma 2] there exists a normal subgroup $G_{1}$ of finite index in $G$, and a subgroup $B$ of $A$, such that $A / B$ is torsion-free, $B^{G_{1}}=B$, and $B$ is a plinth of $G_{1}$. Let $\left\{t_{1}, \ldots, t_{n}\right\}$ be a transversal to $G_{1}$ in $G$. Replacing $\lambda$ by $\prod_{i=1}^{n} \lambda^{t_{i}}$ (non-zero since $k A$ is a domain) we may
assume that $G=G_{1}$.

Now $A=B \times C$, for some subgroup $C$ of $A$. Let

$$
\lambda=\beta_{1}+\sum_{j=2}^{r} \beta_{i} c_{j},
$$

where $\beta_{1}, \ldots, \beta_{r} \in k B$, and $1, c_{2}, \ldots, c_{r}$ are distinct elements of $C$. If necessary, we may replace $\lambda$ by $\lambda c$, for some $c \in C$, to ensure that $\beta_{1} \neq 0$. Let $g \in G$, and write $c_{j}^{8}=b_{j} c_{j}^{\prime}$, for $b_{j} \in B, c_{j}^{\prime} \in C$; so $1, c_{2}^{\prime}, \ldots, c_{r}^{\prime}$ are distinct, and

$$
\lambda^{\mathrm{g}}=\beta_{1}^{\mathrm{g}}+\sum_{j=2}^{r} \beta_{i}^{\prime} c_{j}^{\prime}
$$

with $\beta_{i}^{\prime} \in k B$.

By the induction hypothesis, there exists a maximal ideal $T$ of $k B$ such that $\beta_{1}^{g} \notin T$ for all $g \in G$. Let $D=T^{+}$; so $B / D$ is finite by the Nullstellensatz, since $k$ is a finite field. Hence, $|A: D C|<\infty$. Since $D$ has only finitely many $G$-conjugates, and $\operatorname{dim}_{k}(k B / T)<\infty$, we also have $\left|G: C_{G}(k B / T)\right|<\infty$. Thus $H=N_{G}(D C) \cap C_{G}(k B / T)$ has finite index in $G$. Let $\left\{g_{1}, \ldots, g_{m}\right\}$ be a transversal to $H$ in $G$, and put $\mu=\prod_{i=1}^{m} \lambda^{g_{i}}$.

Put $K=k B / T$, an absolute field, and define $\pi: k A \rightarrow K(A / B)$ by $\pi\left(\sum \beta_{c} c\right)=$ $\sum\left(\beta_{c}+T\right) B c\left(\beta_{c} \in k B\right)$. It is easily seen that $\pi$ is a well-defined ring epimorphism with kernel $T k A$, and that $\pi$ is an $H$-module map. We claim that $\pi(\mu) \neq 0$. For $\pi(\mu)=$ $\prod_{i} \pi\left(\lambda^{B_{1}}\right)$, and so if $\pi(\mu)=0$ then $\pi\left(\lambda^{\beta_{i}}\right)=0$ for some $i$, since $K(A / B)$ is a domain. That is, $\lambda^{g_{1}} \in T k A$; so from $(1), \beta_{1}^{g_{1}} \in T$, a contradiction. Hence, $\pi(\mu) \neq 0$.

The induction hypothesis now implies that there exists a maximal ideal $L$ of $k A$ such that $\pi(\mu)^{h} \notin \pi(L)$ for all $h \in H$. Hence, $\mu^{h} \notin L$ for all $h \in H$, and so $\lambda^{g} \notin L$ for all $g \in G$, as required.

3. Preliminary lemmas and definitions. Recall that an ideal $I$ of a Noetherian ring $R$ is said to have the (right) $A R$ property if, given a right ideal $E$ of $R$, there exists $n \geq 1$ such that $E \cap I^{n} \subseteq E I$. This amounts to requiring that every finitely generated essential extension of an $R$-module $M$ for which $I \subseteq \operatorname{Ann}(M)$ is annihilated by some power of $I$; see [1, Lemma 1]. A related concept which we also need is that of a clan; a prime ideal $P$ of $R$ belongs to a clan if $P$ belongs to a finite set $\left\{P_{1}, P_{2}, \ldots, P_{n}\right\}$ of mutually incomparable prime ideals of $R$ such that the chieftain $N=\bigcap_{i=1}^{n} P_{i}$ of $P$ is localisable, and $N_{N}$ has the 
AR property in $R_{N}$. The definition and basic properties of clans can be found in [9]. If $P$ is maximal then $N$ is semimaximal, by the proof of [9, Theorem 5]. If $R / P_{i}$ is Artinian, $1 \leq i \leq n$, then [8, Theorem 4.5] shows that $N$ itself has the AR property; in fact $N$ is simply the largest ideal which annihilates every chief factor of the injective hulls of $R / P_{i}$, for $i=1, \ldots, n$.

LEMMA 3.1. Let $G$ be a polycyclic-by-finite group, let $J$ be an absolutely Hilbert ring, and let $I$ be an ideal of $J G$ with the AR property. Suppose that $M$ is a finitely generated $J G$-module, all of whose irreducible images are annihilated by I. Then I annihilates every chief factor of $M$.

Proof. Suppose the result is false, and let $U / V$ be a chief factor of $M$, with $U I \nsubseteq V$. Let $V$ be chosen maximal such that $U / V$ is a counterexample. Then clearly $U / V$ is essential in $M / V$. It follows from the main result of [7] that $M / V$ is Artinian. We can thus extract from $M / V$ the uniform submodule $X$, with a short exact sequence

$$
0 \rightarrow U / V \rightarrow X \rightarrow Y \rightarrow 0
$$

where $Y$ is irreducible, and $Y I=0$.

Let $P=\operatorname{Ann}(U / V), Q=\operatorname{Ann}(Y)$. By [3, Theorem 4.5], $P$ belongs to a clan; let $N$ be its chieftain. As noted above, $N \subseteq Q$. It follows from [9, Theorem 5] that clan $(Q)=$ clan $(P)$, so that $N$ is the chieftain of $Q$. Since $I$ has the AR property and $I \subseteq Q, I$ annihilates every chief factor in the injective hull of $R / Q$, and moreover every chief factor in the injective hulls of these chief factors is killed by $I$. It follows that $I \subseteq N$. This completes the proof.

The proof of the next result is left to the reader.

LEMMA 3.2. The product of finitely many ideals with the AR property has the AR property.

We refer the reader to [5] for the definition and basic properties of the Krull dimension. Recall that the dimension of a finitely generated right module $M$ over the Noetherian ring $R$ will be denoted by $\mathrm{k}-\operatorname{dim}_{R}(M)$, or simply by $\mathrm{k}-\operatorname{dim}(M)$. If $M$ is a left module, its left Krull dimension will be denoted by $1-\operatorname{dim}_{R}(M)$, or l-dim $(M)$. The ring $R$ is said to be $K$-homogeneous if all its non-zero ideals have the same right Krull dimension.

LEMMA 3.3. Let $J$ be an absolutely Hilbert ring, $G$ a polycyclic-by-finite group.

(i) If $I$ is an ideal of $J G$, and $H$ is a normal subgroup of finite index in $G$, then $\mathrm{k}-\operatorname{dim}_{J G}(J G / I)=\mathrm{k}-\operatorname{dim}_{J H}(J H / I \cap J H)$.

(ii) If $T, I$ are ideals of $R G$ with $T \subseteq I$ then $\mathrm{k}-\operatorname{dim}(I / T)=1-\operatorname{dim}(I / T)$.

Proof. (i) By [16, Lemma 8], $\mathrm{k}-\operatorname{dim}_{J G}(J G / I)=\mathrm{k}-\operatorname{dim}_{R H}(J G / I)$. (This is proved in [16] under the extra hypothesis that $k-\operatorname{dim}_{J}(J)<\infty$, but this is unnecessary.) It follows that

$$
\begin{aligned}
\mathrm{k}-\operatorname{dim}_{J G}(J G / I) & \geq \mathrm{k}-\operatorname{dim}_{J H}(J H+I / I) \\
& =\mathrm{k}-\operatorname{dim}_{J H}(J H / I \cap J H) .
\end{aligned}
$$


For the reverse inequality, it suffices to note that $J G / I$ is a finitely generated $(J H / I \cap J H)$ module.

(ii) Suppose the result is false, and choose $T$ as large as possible such that there is an ideal $I$ containing $T$ for which the stated equality fails. Then it is easily seen that, for all ideals $X$ of $J G$ such that $T \varsubsetneqq X, T \varsubsetneqq X \cap I$. From [2, Theorems 4.2 and 6.3], it follows that

$$
\mathrm{k}-\operatorname{dim}_{I G}(I / T)=\mathrm{k}-\operatorname{dim}_{J G}(J G / T),
$$

and similarly,

$$
1-\operatorname{dim}_{J G}(I / T)=1-\operatorname{dim}_{J G}(J G / T) \text {. }
$$

However, by [3, Theorem 3.14],

$$
\mathrm{k}-\operatorname{dim}(J G / T)=1-\operatorname{dim}(J G / T) \text {. }
$$

Hence, $\mathrm{k}-\operatorname{dim}(I / T)=1-\operatorname{dim}(I / T)$, contradicting the choice of $T$. This completes the proof.

4. Proofs of the main results. It is convenient to make an ad hoc definition to describe a type of group which will appear in the course of the proof. Thus, a polycyclicby-finite group $G$ is said to satisfy hypothesis $(*)$ if the following hold:

(a) $G$ is torsion free;

(b) $G$ is orbitally sound (see $[14, \S 1.3]$ );

(c) the FC-subgroup $\Delta(G)=\left\{g \in G:\left|G: C_{G}(g)\right|<\infty\right\}$ is central in $G$;

(d) $G$ contains a series of normal subgroups

$$
1=G_{0} \subset G_{1} \subset \ldots \subset G_{n}=G
$$

such that $G_{i} / G_{i-1}$ is a plinth for $G / G_{i-1}$, for $1 \leq i \leq n$.

We now show that such groups will arise.

LEMMA 4.1. If $G$ is a polycyclic-by-finite group, there exists a normal subgroup $H$ of finite index in $G$ which satisfies (*).

Proof. It is easily checked that the class of groups satisfying each of (a)-(d) is closed under passage to subgroups of finite index, so it suffices to find a subgroup of finite index in $G$ satisfying each property. For (a), this follows from [12, Lemma 10.2.5]. For (b), use [14, Theorem C2]. Since $\Delta(G)$ is finitely generated, $\Delta(C)$ is central, where $C=C_{G}(\Delta(G))$, and this gives (c). Finally, for (d), we use [12, Lemma 12.1.4], and induction.

We shall prove Theorems A and B in tandem. Recall that $J$ is an absolutely Hilbert domain, $G$ is a polycyclic-by-finite group, $M$ is a finitely generated $J G$-module, with annihilator $I$, and

$$
T_{G}(M)=\bigcap\{\operatorname{Ann}(X): X \text { an irreducible image of } M\} .
$$

Then Theorem A states that $J G / I$ and $J G / T_{G}(M)$ have the same Krull dimension.

Deduction of Theorem $B$ from Theorem $A$. We are given an ideal $K$, with $I \subseteq K \subseteq$ $T_{G}(M)$, such that $K / I$ has the AR property; we have to show that $K / I$ is nilpotent. There 
exists a finite chain

$$
0=M_{0} \subset M_{1} \subset \ldots \subset M_{n}=M
$$

of submodules of $M$, such that $\operatorname{Ann}\left(M_{i} / M_{i-1}\right)$ is a prime ideal, $1 \leq i \leq n$, since $M$ is a Noetherian $J G$-module. By Lemma 3.1, $K$ kills every chief factor of $M$; in particular, $K \subseteq T_{G}\left(M_{i} / M_{i-1}\right)$, for $1 \leq i \leq n$. By Theorem A, $T_{G}\left(M_{i} / M_{i-1}\right)=\operatorname{Ann}\left(M_{i} / M_{i-1}\right)$. Hence $K^{n} \subseteq \operatorname{Ann}(M)$, as required.

Proof of Theorem A. We break the argument into a sequence of steps.

Step I: If $H$ is a normal subgroup of finite index in $G$, and the result is true for $J H$, then it is true for $J G$. To see this, view $M$ as a $J H$-module. Now $T_{H}(M) \supseteq T_{G}(M) \cap J H$. For, let $M / V=Y$ be a simple $J H$-module. If $X$ is a simple image of the $J G$-module $M / \bigcap_{\mathrm{g} \in G} V g$ then $X T_{G}(M)=0$, and so $E\left(T_{G}(M) \cap J H\right)=0$, for any simple $\mathbb{Z} H$-module $E$ which is an image of $X$. However, $E \cong M / V g$, for some $g \in G$, and so, since $\left(T_{G}(M) \cap J H\right)^{\mathrm{g}}=$ $\left(T_{G}(M) \cap J H\right), Y\left(T_{G}(M) \cap J H\right)=0$.

Now $A_{n_{J H}}(M)=I \cap J H$, and, by hypothesis,

$$
\mathrm{k}-\operatorname{dim}_{J H}(J H / I \cap J H)=\mathrm{k}-\operatorname{dim}_{J H}\left(J H / T_{\mathrm{H}}(M)\right) .
$$

Thus, by the above, and Lemma 3.3(i),

$$
\begin{aligned}
\mathrm{k}-\operatorname{dim}_{J G}\left(J G / T_{G}(M)\right) & =\mathrm{k}-\operatorname{dim}_{J H}\left(J H / T_{G}(M) \cap J H\right) \\
& \geq \mathrm{k}-\operatorname{dim}_{J H}\left(J H / T_{H}(M)\right) \\
& =\mathrm{k}-\operatorname{dim}_{J H}(J H / I \cap J H) \\
& =\mathrm{k}-\operatorname{dim}_{J G}(J G / I) .
\end{aligned}
$$

Since $T_{G}(M) \supseteq I$, this proves Step $I$.

Step II: The induction set-up. We argue by induction on the Hirsch number $h(G)$ of the group $G$; that is, the number of infinite cyclic factors occurring in a subnormal series for $G$ with factors cyclic or finite. If $h(G)=0$ then $G$ is finite and, by Step I, we may assume that $G=1$. An argument of the type used in deducing Theorem B shows that $M$ may be assumed to be cyclic with prime annihilator $P$; that is, $M \cong J / P$. Since $J$ is a Hilbert ring, $P$ is semisimple, and the result is proved.

Suppose then that $h(G)>0$, and that the result is known for groups of smaller Hirsch number. We suppose that the $J G$-module $M$ affords a counterexample to the theorem, and aim for a contradiction. Since $J G$ is Noetherian, it may be assumed that

(i) $\mathrm{k}-\operatorname{dim}\left(J G / T_{G}(\bar{M})\right)=\mathrm{k}-\operatorname{dim}(J G / \operatorname{Ann}(\bar{M}))$ for any module $\bar{M}$ whose annihilator strictly contains $I$;

since $M$ is Noetherian, we may also assume that

(ii) $\mathrm{k}-\operatorname{dim}\left(J G / T_{G}(\bar{M})\right)=\mathrm{k}-\operatorname{dim}(J G / \operatorname{Ann}(\bar{M}))$ for all proper factors $\bar{M}$ of $M$.

By Lemma 4.1, there exists a normal subgroup $H$ of finite index in $G$ which satisfies (*). By Step I, there is no loss in assuming that $H=G$, and this we shall henceforth do.

Step III: The ring JG/I is $K$-homogeneous. By [2, Theorems 6.2 and 4.2], and [3, Theorem 3.13], there exist ideals $I_{1}, \ldots, I_{t}$ and $J G$ such that $J G / I_{i}$ is a $\mathrm{K}$-homogeneous 
ring, $1 \leq \mathrm{j} \leq \mathrm{t}$, and $I=\bigcap_{j=1}^{\mathrm{t}} I_{j}$. Let $\mathrm{k}-\operatorname{dim}(J G / I)=\alpha$, and suppose that $J G / I$ is not $\mathrm{K}$ homogeneous. Then $t>1$, and there exists $r, 1 \leq r<t$, such that $\mathrm{k}-\operatorname{dim}\left(J G / I_{j}\right)=\alpha$ for $1 \leq j \leq r, \quad$ while $\mathrm{k}-\operatorname{dim}\left(J G / I_{j}\right)<\alpha$ for $j>r . \quad$ Put $A=\bigcap_{i=1}^{r} I_{j}, \quad B=\bigcap_{j=r+1}^{t} I_{j}$, so that
$\mathrm{k}-\operatorname{dim}(J G / A)=\alpha$, and $\mathrm{k}-\operatorname{dim}(J G / B)<\alpha$.

By the induction hypothesis on $I$,

$$
\mathrm{k}-\operatorname{dim}\left(J G / T_{G}(M / M A)\right)=\mathrm{k}-\operatorname{dim}(J G / \operatorname{Ann}(M / M A)) \text {. }
$$

Let $Y=\operatorname{Ann}(M / M A)$, so that $M Y B \subseteq M A B \subseteq M I=0$. Thus, $Y \subseteq 1-A n n(B / I)$. Suppose that $\mathrm{k}-\operatorname{dim}(J G / Y)<\mathrm{k}-\operatorname{dim}(J G / A)$. Then

$$
1-\operatorname{dim}(B / I) \leq \mathrm{k}-\operatorname{dim}(J G / Y)<\alpha .
$$

By Lemma 3.3(ii), this yields $\mathrm{k}-\operatorname{dim}(B / I)<\alpha$, and since $\mathrm{k}-\operatorname{dim}(J G / B)<\alpha$, this is a contradiction. Hence,

$$
\mathrm{k}-\operatorname{dim}(J G / \operatorname{Ann}(M / M A))=\alpha .
$$

Since $T_{G}(M)=T_{G}(M / M A) \cap T_{G}(M / M B)$, and $I \subseteq T_{G}(M)$, (3) and (4) contradict our choice of $M$ as a counterexample. Therefore $J G / I$ is $K$-homogeneous.

Step $I V$ : The ideal $I$ is prime. Suppose not, and let $A, B$ be ideals of $J G$, with $I \varsubsetneqq A$, $I \subsetneq B$, and $A B \subseteq I$. There is no loss in assuming that $A=1-\operatorname{Ann}(B)$, so that $\operatorname{Ann}(M / M A)=A$. By Lemma 3.3(ii) and Step III,

$$
\mathrm{k}-\operatorname{dim}(J G / A)=1-\operatorname{dim}(J G / A)=1-\operatorname{dim}(B / A)=\alpha .
$$

Hence, using the induction hypothesis on $I$, we have

$$
\mathrm{k}-\operatorname{dim}\left(J G / T_{G}(M / M A)\right)=\mathrm{k}-\operatorname{dim}(J G / A)=\alpha .
$$

Since $T_{G}(M)=T_{G}(M / M A) \cap T_{G}(M / M B)$, it follows that $\mathrm{k}-\operatorname{dim}\left(J G / T_{G}(M)\right)=\alpha$, contradicting the choice of $M$. Therefore, $I$ is prime.

Step $V: M$ is not torsion free over a certain ring. Since $I$ is prime, $I \cap J$ is a prime ideal of $J$. Factoring if necessary by $(I \cap J) G$, we may thus assume that $I \cap J=0$. If $I^{+} \neq 1$, it is infinite, since $G$ is torsion free, and the result would follow by induction on $h(G)$, since $J G / i G \cong J\left(G / I^{+}\right)$. Hence $I^{+}=1$. Recall that the FC-subgroup $C$ of $G$ is central. Since $G$ is orbitally sound, $I=(I \cap J C) J G$ by $[14$, Theorem C1].

Let $T=T_{G}(M)$; since $I$ is prime, the assumption that $M$ is a counterexample to Theorem A amounts to saying that $T \supsetneqq I$. We shall show that this leads to a contradiction. Note that $T$ is a semiprime ideal. Let $Q_{1}, \ldots, Q_{t}$ be prime ideals of $J G$ such that $T=\bigcap_{i=1}^{\prime} Q_{i}$. Let $r$ be such that $Q_{i}^{+}$is infinite for all $i=1, \ldots, r$, while $Q_{i}^{+}=1$ for $r+1 \leq i \leq t$.

For $0 \leq i \leq r$, define $E_{i}$ by: $E_{0}=C$; for $i \geq 1, E_{i}=E_{i-1}$ if $H_{i}=Q_{i}^{+} \cap E_{i-1} \neq 1$, and, if $Q_{i}^{+} \cap E_{i-1}=1, E_{i}=E_{i-1} H_{i}$, where $H_{i} \subseteq Q_{i}^{+}$is a nontrivial Abelian normal subgroup of $G$. Put $E=E_{r}$, so $E \triangleleft G, E=C \times H$, say. For $r+1 \leq i \leq t, Q_{i}=\left(Q_{i} \cap J C\right) J G$, and so, since $(I \cap J C) J G=I \varsubsetneqq T \subseteq Q_{i}$, there exists $\mu_{i} \in\left(Q_{i} \cap J C\right) \backslash(I \cap J C)$. For $1 \leq i \leq r$, choose $h_{i} \in H_{i}$, 
$h_{i} \neq 1$. Note that $h_{i}-1 \notin I \cap J E$, since $I^{+}=1$. Consider the element $\beta=\prod_{i=1}^{r}\left(h_{i}-1\right) \prod_{i=r+1}^{t} \mu_{i}$ of $J E$. Clearly, $\beta \in \bigcap_{i=1}^{t} Q_{i}=T$. Since $C$ is central, $I \cap J C$ is a prime ideal of $J C$, and so $I \cap J E=(I \cap J C) J E$ is a prime ideal of $J E$. Thus, it follows that $\beta \notin I \cap J E$.

Let $S=J E / I \cap J E$. If $M$ is torsion free as an $S$-module, there exists a free $S$ submodule $F$ of $M$, and $\lambda \in J E \backslash(I \cap J E)$, such that $M / F$ is $\lambda^{G}$-torsion by [13, §3]. Let $L$ be a maximal ideal of $J E$ containing $I \cap J E$, and put $L^{0}=\bigcap_{\mathrm{g} \in \mathrm{G}} L^{\mathrm{g}}$, so $L^{0}$ is semimaximal. If $B=\prod_{i=1}^{r} \mathfrak{h}_{i} E \prod_{i=r+1}^{t} \mu_{i} \nsubseteq L$, then $J E=B+L^{0}$. Since $B \subseteq T, B$ kills every simple image of $M$, and so $M=M^{0} L$. Thus every maximal ideal $L$ of $J E$ which contains $I \cap J E$ and with $B \nsubseteq L$ must contain a conjugate of $\lambda$, as in [13, Corollary C1]. Since $\beta \in B$ it follows that every maximal ideal of $J E$ which contains $I \cap J E$ also contains a conjugate of $\lambda \beta$. But since $\lambda \beta \notin I \cap J E$, and $J E / I \cap J E \cong(J C / I \cap J C) H$, this contradicts Theorem 2.1. Hence $M$ is not a torsion free $S$-module.

Step VI: Completion of the proof. Let the ideal $P$ of $S$ be maximal among annihilators of non-zero $S$-submodules of $M$, and let $U=\{m \in M: m P=0\}, V=U J G$, and $N=N_{G}(P)$, so that $E \subseteq N$. If $X$ is a transversal to $N$ in $G$ then, by [13, Lemma 3], $U$ is a Noetherian $J N$-module, and $V=\sum_{x \in X}^{\oplus} U x$.

Let $U / Y$ be a simple $J N$-module. Then there exists a simple $J G$-module $V / W$ such that $U / Y$ embeds in $V / W$; just choose a maximal submodule $W$ containing $Y G$. For $1 \leq i \leq r$, the ideal $h_{i} G$ of $J G$ has the AR property by [12, Theorem 11.2.14], and the same applies to the ideal $\left(\prod_{i=r+1}^{t} \mu_{i}\right) J G$ by [12, Theorem 11.2.2], since $\prod_{i=r+1}^{t} \mu_{i}$ is central. Hence, $B G=\prod_{i} \mathfrak{h}_{i} G \prod_{i} \mu_{i}$ has the AR property by Lemma 3.2. Since $B G \subseteq T$, it follows from Lemma 3.1 that $V(B G) \subseteq W$. From this, we obtain $U(B N) \subseteq Y$. That is, the ideal $B N$ of $J N$ annihilates every simple image of $U$.

Now suppose that (a) $|G: N|=\infty$. Then, by induction on the Hirsch number, Theorems $\mathrm{A}$ and $\mathrm{B}$ are true for $J N$-modules. Thus, by Theorem $\mathrm{B}$ applied to $U$, there exists $k_{1} \geq 1$ such that $U(B N)^{k_{1}}=0$. Since $B$ is $G$-invariant, $V(B G)^{k_{1}}=0$. Now, since $I$ is prime, and $T_{G}(M / V) \supseteq T_{G}(M)$, and since Theorem $A$ is true for $M / V$, we must have $\operatorname{Ann}_{J G}(M / V) \supsetneqq I$. Hence, Theorem B applies to $M / V$, and there exists $k_{2} \geq 1$ such that $M(B G)^{k_{2}} \subseteq V$. Therefore $M(B G)^{k_{1}+k_{2}}=0$, and so, since $I$ is prime, $B G \subseteq I$, a contradiction. This completes the proof in case (a).

Suppose that (b) $|G: N|<\infty$. Then there exist only finitely many $G$-conjugates of $P=P_{1}, P_{2}, \ldots, P_{m}$. Set $Q=(I \cap J E)+\prod_{i=1}^{m} P_{i} \triangleleft J E$. Since $I \cap J E$ is prime and $G$-invariant, and $P \supsetneqq I \cap J E$, it follows that $Q \supsetneqq I \cap J E$. Let $\bar{U}=\{m \in M: m Q=0\}$. Since $Q$ is $G$-invariant, $\bar{U}$ is a $J G$-submodule, and $U \subseteq \bar{U}$, so that $\bar{U} \neq 0$. Once again, the maximality of $I$ ensures the validity of Theorems $\mathrm{A}$ and $\mathrm{B}$ for $\bar{U}$, so, as above, there exists $k_{1} \geq 1$ such that $\bar{U}(B G)^{k_{1}}=0$. Similarly, there exists $k_{2} \geq 1$ such that $M(B G)^{k_{2}} \subseteq \bar{U}$, and as before we deduce that $B G \subseteq I$, a contradiction. This completes the proof of the theorem. 
The following corollary answers the question raised in [13, p. 310] in the affirmative.

Corollary C. Let $J, G$ and $M$ be as in Theorem A. If $H$ is a normal nilpotent subgroup of $G$, and $T$ is an ideal of $J H$ which annihilates every irreducible image of $M$, then $M T^{n}=0$ for some $n \geq 1$.

Proof. The stated hypotheses apply to every $G$-conjugate of $T$, so there is no loss in assuming that $T^{G}=T$. Since $H$ is nilpotent, $T$ is a polycentral ideal of $J H[15$, Theorem C]. By [12, Theorem 11.2.9], it follows that the ideal $T G$ of $J G$ has the AR property. The corollary now follows from Theorem $B$.

The following generalisation of $[13$, Corollary B] can be proved by the same argument used to deduce Theorem B.

Corollary D. Let $J, G$ and $M$ be as in Theorem A. If the ideal $T$ of $J G$ annihilates every chief factor of $M$, then there exists $n \geq 1$ such that $M T^{n}=0$.

5. Concluding remarks. It would be interesting to know the extent to which the hypothesis that $J$ is absolutely Hilbert is necessary for Theorems A and B. Since $J$ is an image of $J G$, the conclusion of Theorem A clearly implies that $J$ must be a Hilbert ring, but it seems plausible that this condition might also be sufficient.

Ian Musson has shown that the solvable Lie algebra $L=\langle x, y:[x, y]=x\rangle$ over any field $K$ of characteristic zero has enveloping algebra $R$ which admits a finitely generated uniserial module $M$ of Krull dimension one [11]. Indeed, the unique irreducible image $\bar{M}$ of $M$ has dimension one over $K$, and so $R / \operatorname{Ann}(\bar{M})$ is Artinian. Since $R$ is a Hilbert ring [4] this example shows that Theorem $A$ is not valid for arbitrary Hilbert rings.

\section{REFERENCES}

1. M. Boratyński, A change of rings theorem and the Artin-Rees property, Proc. Amer. Math. Soc. 53 (1975), 307-310.

2. K. A. Brown, Module extensions over Noetherian rings, to appear in J. Algebra.

3. K. A. Brown, T. H. Lenagan and J. T. Stafford, $K$-theory and stable structure of some Noetherian group rings, Proc. London Math. Soc., (3) 42 (1981), 193-230.

4. M. Duflo, Certaines algèbres de type fini sont des algèbres de Jacobson, J. Algebra 27 (1973), 358-365.

5. R. Gordon and J. C. Robson, Krull dimension, Mem. Amer. Math. Soc. 133 (1973).

6. D. L. Harper, Primitive irreducible representations of polycyclic groups, Ph.D. dissertation, Queen's College, Cambridge (1977).

7. A. V. Jategaonkar, Integral group rings of polycyclic-by-finite groups, J. Pure Appl. Algebra 4 (1974), 337-343.

8. A. V. Jategaonkar, Injective modules and localization in noncommutative Noetherian rings, Trans. Amer. Math. Soc. 190 (1974), 109-123.

9. B. J. Müller, Localisation in non-commutative Noetherian rings, Canad. J. Math. 28 (1976), 600-610.

10. 1. M. Musson, Injective models for group rings of polycyclic groups II, Quart, J. Math. (2) 31 (1980), 449-466.

11. I. M. Musson, Uniserial modules over enveloping algebras, unpublished note. 
12. D. S. Passman, The algebraic structure of group rings (Interscience, 1977).

13. J. E. Roseblade, Group rings of polycyclic groups, J. Pure Appl. Algebra 3 (1973), 307-328.

14. J. E. Roseblade, Prime ideals in group rings of polycyclic groups, Proc. London Math. Soc. (3) 36 (1978), 385-447.

15. J. E. Roseblade and P. F. Smith, A note on hypercentral group rings, J. London Math. Soc. (2) 13 (1976), 183-190.

16. D. Segal, On the residual simplicity of certain modules, Proc. London Math. Soc. (3) 34 (1977), 327-353.

Department of Mathematics

UNIVERSITY OF GLASGOW

GLASGOW

SCOTLAND

G12 8QW 